\author{
I J M I \\ International Journal of Machine Intelligence \\ ISSN: 0975-2927 \& E-ISSN: 0975-9166, Volume 3, Issue 3, 2011, pp-142-145 \\ Available online at http://www.bioinfo.in/contents.php?id=31
}

\title{
POLLY CLINIC INQUIRY SYSTEM USING IVR IN MARATHI LANGUAGE
}

SANTOSH GAIKWAD*, BHARTI GAWALI, MEHROTRA S.C.

Department of computer Science \& Information Technology,Dr.Babasaheb Ambedkar Marathwada University Aurangabad.

*Corresponding Author: Email-santosh.gaikwadcsit@gmail.com,bharti_rokade@yahoo.co.in

Received: September 29, 2011; Accepted: November 03, 2011

\begin{abstract}
A convenient and user -friendly interface for information retrieval through computer is an important issue. Speech being natural mode of communication among human beings has the potential of being a primary as well as convenient mode of interaction with computer. This is a good reason for building systems that communicate with users via speech. Marathi is one of the most widely used language is Maharashtra. An interesting domain for such speech understanding systems is information dialogs where the user wants to get some information by asking the system which takes the role of an "Intelligent Machine" in the field of interest. Speech recognition having much more application in health sector In order to make such a communication process possible we explore the new approach of Polly Clinic Inquiry System using Marathi Speech Recognition.
\end{abstract}

Keywords - Speech Recognition, polyclinic, IVR, Marathi

\section{Introduction}

Speech Recognition is technologies of particular interest, for their support of direct communication between humans and computers, through a communications mode. As Computer become available everywhere, researchers must create an interest to build a real time system that are used for simple user. As such interface must accept verbal input, since speech is the most natural, efficient, flexible, and economical form of human communication. The most obvious barrier to speech input is automatic speech recognition, which converts the speech signal to discrete symbols (usually a set of words). This is a challenging problem, with well-defined applications such as dictation and simple data entry. The success of such a system is measured by its ability to understand the input and take appropriate action, rather than by word accuracy alone. Speech understanding is an active process that uses many different sources of knowledge, some of which are deeply embedded in the linguistic competence of the speaker and the listener.

Perhaps the most important challenge to the development of speech understanding systems is the integration of speech recognition and natural-language processing. Researchers in each discipline must determine how their systems can best exchange and use information. In some cases, system designs might require fundamental changes. It is possible that a Computer Speech interface permit spoken dialog in native language. The advantages of this is that majority of people can be benefit from this technology. When they are able to interact interact with computer in their native language. There is various application of computer speech interface Automated attendant,ACD Systems, Alerts \& Reminders ,Card Activations , Hotline \& helpdesk. The famous application of speech recognition is currently IVRS. When you call any companies there is reply form instead of talking live person, caller is greeted by a pre recorded massage with technology understanding word the caller speaks the selection using the phone keybord.whe implemented correctly ,an IVRS can help people transaction to get answer over the phone faster than they would by speaking to a live representative. The benefit of machine is quick action and machine doesn't tire any time. For example, appreciate the ability to quickly check the balance on bank account, flight reservation, and train reservation.

Although there is no evidence of consistent progress comparable to that machine generated speech .Richard fatehchand [8] was able to write that there was in 1960 no machine capable of dealing with Continuous speech. Lindgren[9] five years latest was able to say same. It is possible to repeat the statement, the scheme developed at Standford by Vicens [10] deals with a robot application based on connected word recognition. Actually the success of Continuous speech recognition is started from 1997 in Product of dragon System.But this system is also some limitation speaker dependant.

The Speech recognition is also used is health application .the first application of speech Recognition is First use of speech recognition in the field of radiology in 1981.same 2008 the application of IBM in the reading pathology report is having Mean accuracy rate of reading pathology reports, using IBM Via Voice Pro software - 93.6\% compared to human transcription at $99.6 \%$.

Shiffman S, Lane CD, Johnson KB, Fagan LM in 1992 develops a continuous-speech interface for Quick Medical Reference (QMR), which allows physicians to input spoken descriptions of physical-examination findings, or observations [12]. 
J Am Med Inform Assoc in 1995 builds the develop a continuous-speech interface that allows flexible input of clinical findings into a medical diagnostic application.[11]

Department of Computing, The Nottingham Trent University, Burton Street, UK in 2002 development of a speech interface to a virtual world and to consider its relevance for disabled users. [13]

Marathi is an Indo Aryan language spoken by the Marathi people of western India (Maharshtrians). It serves as the official language of the state of Maharashtra, with roughly ninety million fluent speakers worldwide. Lots of research is already started in English, Bengali, Nepali but little bit attention to Marathi Language, and speech computer interface using Marathi language is one of the challenging areas. Doctors play very important role in real life. So, we scope toward build Polly Clinic Inquiry System using IVR in Marathi Language.

The rest of Paper is Recognize is as follows .The Creation of Database related to Polly clinic applications presented in section 2. A concept of Polyclinic (Multispecilist Hospital) is presented in section 3.An experimental analysis work presented in section 4 section 5 present result \& Conclusion.

\section{Database Preparation}

For recognizing accurate speech, a computer should be trained with speech data. In that speech data we need collection of utterances, so for any speech recognition system speech database has to be built. In speech technology, the Maturity and scientificity of speech database is very important [3].our system is speaker independent means it need large number of data prepared by different group of people age wise and gender wise. Speech database includes the training set and testing set, the process of speech database includes preparations (recording divide, recording software etc.) sentence collection and selection, speaker selection [2]. The generation of Marathi speech database for intelligent system for clinic application using speech recognition is given below. In this application, we maintain the three set of database. One database corresponds to doctor name, second for queries of inquiry about doctor and third with the system call back answer or solution. in collection of database age group speaker selected from ranges 20 to 40. For marinating accuracy we choose subject from different native moth tongue but they speak today marathi. The total number of speaker is 15 out of which 6 were females $\& 9$ were males. Vocublary size of database consist of : 2030 sentences

Database with name of doctor: 50 isolated word.

Database with queries of doctor inquiries $=2$ doctor 16 queries ${ }^{\star} 15$ speaker* 4 utterances $=1920$ sentences

Solution database as sentences: 60 samples.

\section{Acquisition setup}

To achieve a high quality the recording took place in the normal room. the sampling frequency $16000 \mathrm{~Hz}$ In the room temperature. The speaker were seating in front of the direction of microphone with the distance about 12-15 $\mathrm{cm}$. The speech data is collected with the help of
Computerized Speech Lab. The CSL is most advanced analysis system for speech Recognition. It is complete hardware and software system with specification and performance. It is an input Output Recording Device for a PC.

\section{Polly Clinic Inquiry System}

Doctors play very important role in real life. If we have any problem about our health there is need to meet doctor and follow doctor's prescription. In case of ill health we need to visit the clinic and get appointment of doctor to solve our problem.

A polyclinic, or policlinic, is a place where a wide range of health care services (including diagnostics) can be obtained. The hospital is also one of the largest clinical industries areas.

Marathi is an Indo Aryan language spoken by the Marathi people of western India (Maharshtrians). It serves as the official language of the state of Maharashtra, with roughly ninety million fluent speakers worldwide. Marathi ranks 4th in India with respect to the number of people who claim it as their primary language. Along with Bengali, Marathi is the oldest of the regional literatures in IndoAryan languages, dating from about 1000 AD. Marathi is at least fifteen hundred years old, and derives its grammar and syntax from Pali and Prakrit.

In real life there is need implement a system that are helpful for progress growth of Hospitality .In polyclinic countable in hundred doctors are available day to day so, it is not possible to keep in mind that day of doctor as per specialty as well as fees. for that purpose we call to hospital there is receptionist available he/she give information waiting some time .all work are not handling receptionist so, we build an Polyclinic Inquiry system using speech recognition in Marathi Language. The Proposed Working Model of Polyclinic Inquiry System is shown in Figure 1. The proposed PCIS model will be advantages to farmer as it is provide quick solution of query related to doctor inquiry on 24/7 basis.

\section{Working of PCIS}

The unique point of voice interface is the ability to access an information system remotely.

The ubiquitous telecommunication system enables one to access information of an "Anytime anywhere". The proposed working model of PCIS is shown in figure 1 Computer Telephonic card will be used to set up data collection envirment. For working of PCIS system the Interface of CTI card and Computer can be created using PHP -asterisk function [1]. Once the any user call, working of PCIS system starts, which is as follows...

a) Call will connect to PCIS.

b) The System will prompt user to tell the name of Doctor.

c) The System will prompt user to tell the query of inquiry about doctor.

d) PCIS System Search the Keyword form sentence \& find out features of that keywords.

e) The Searching will be communicated to the User. 
f) From Searching Keyword sentence is Recognize.

g) Depending on recognized query the Voice Response from PCIS revent back to user.

h) The PCIS will prompt for continuation, if user want to continue with other symptoms the system will start by step 2 else it will thank the user and terminate.

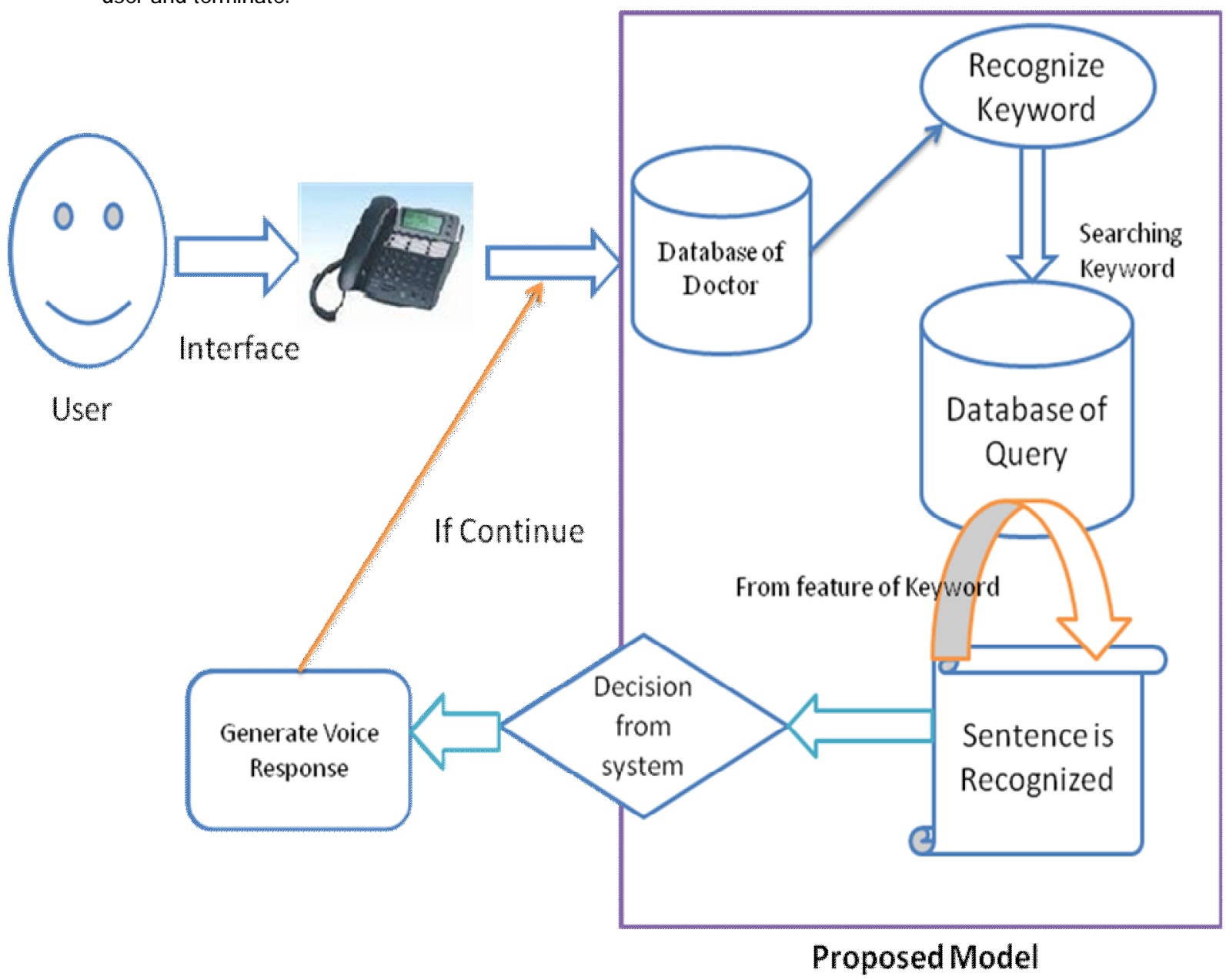

Fig. 1- Proposed Working Model of Polyclinic Inquiry System

\section{Experimental Analysis}

The Experiment for testing Speech Recognition for Polyclinic Inquiry system is based on Speech Recognition. Actually this process is two ways communication i.e. Input Provide to PCIS System is provide using Telephone. In speech recognition accuracy is directly proportional to Vocabulary of Training Database. In this system we Train system as 1000 sentences and 50 isolated word. In this PCIS the query any user is given to the system as a input for recognition .The time for training comes to 4 minute fifty five second, where for testing (recognition) and relevant answer from system is one minutes for 670 continuous sentences. The process is identification of keywords from query and recognizing the sentences The primary performance of system used was percent correct which is defined as

$$
\frac{N-D-S}{N} * 100 \%
$$

\%Word Correct=

Where N, S, and D denotes the total no. of word in the test data, Number of substitution error, and deletion error respectively. Simmilarly, one can evaluate the performance of system at the sentence level. We define $\%$ sentence correct as 100 times the ratio of number of complete sentence organized correctly to the total number of sentences in the test collection. $\mathrm{H}$ is Percentage Correctly Recognize is $\mathrm{H}=\mathrm{N}-(\mathrm{S}+\mathrm{D})$

Table 1: Response of system on the basic Classification of Gender

\begin{tabular}{|l|l|l|l|l|}
\hline Sr.No & Gender & $\begin{array}{l}\text { Sentences } \\
\text { Passed }\end{array}$ & $\begin{array}{l}\text { Sentences } \\
\text { Recognize }\end{array}$ & Accuracy \\
\hline 1 & Male & 130 & 112 & $92 . \%$ \\
\hline 2 & Female & 100 & 90 & $90 \%$ \\
\hline
\end{tabular}


Table 2: Response of system on the basic Classification of age group

\begin{tabular}{|l|l|l|l|l|}
\hline Sr.No & $\begin{array}{l}\text { Age } \\
\text { Group }\end{array}$ & $\begin{array}{l}\text { Sentences } \\
\text { Passed }\end{array}$ & $\begin{array}{l}\text { Sentences } \\
\text { Recognize }\end{array}$ & Accuracy \\
\hline 1 & $20-25$ & 200 & 176 & $87 . \%$ \\
\hline 2 & $25-30$ & 100 & 90 & $90 \%$ \\
\hline 3 & $35-40$ & 50 & 46 & $92 \%$ \\
\hline
\end{tabular}

For computing overall accuracy, the overall accuracy of System is $88 \%$

\section{Complexity of System}

The size of vocabulary of a speech recognition system affects the complexity, processing requirements and the accuracy of the system [5].the complexity of system is calculated in term of time and money. We already mention that the size of vocabulary is training is 1000 sentences so it affect on time of relevant answer from system also we tested all experiment on general computer., but it will be improve using the high system configuration, and earliest version of software's. The prize of system with inbuilt CTI card is average 50,000 so, that is easily available in polyclinic. The system is update monthly. So, there is no extra effort for maintenance.

\section{Result \& Conclusion}

This paper Present a Creation of Large Vocabulary Continuous Speech Database respect to Polyclinic Inquiry system .when tested system it has been found that recognition rate Comes To be $88 \%$.we tried to use the Created database for the application prototyping Polly clinic Inquiry System. This application should be valuable for training speaker independent Continuous speech recognition System for Marathi Language as well as human Machine interface using Speech. This work can be extended in various sector of Medical

\section{Acknowledgment}

The authors would like to thank the university authorities for providing infrastructure to carry out experiment. This work has been supported by DST under Fast Track Scheme entitled as "Design and Development of Marathi Speech Interface System".

\section{References}

[1] A Function of PHP for CTI Available on [online] http://phpagi.sourceforge.net/phpagi2/docs/ele mentindex_phpAGI.html

[2] Samudravijay K. Speech input/output systems in Hindi. Tata Institute of Fundamental Reserch ,Homi Bhabha road,Mumbai 400005

[3] Santosh Gaikwad, Bharti Gawali, PravinYannawar (2010) International Jounal of ComputerApplication, volume10/number3/14621976.

[4] Bharti Gawali,santosh gaikwad,Pravin Yannawar,Suresh Mehrotra (2010) ACEEE Dec 2010.

[5] http://www.speech.cs.cmu.edu/comp.speech/Se ction6/Q6.1.html

[6] Speech recognition and its Clinical Appliction, Thankam Thyvalikakath, MDS Center for Biomedical Informatics University of Pittsburgh

[7] David R. Hill. Man-Machine interaction Using Speech, Department of Mathmatics \& Computing science, The University of calagary,Alberta, Canada

[8] Fatehchand R. (1960) Advan. Computers 1, 193-229.

[9] Lindgrcn N. (1965) IEEE Spectrum 2, No. 3, 114-136.

[10] Vicens P. (1969) Aspects of speech recognition by computer, Ph.D. Dissertation, CS 127,Dept. of Computer Sci., Stanford Univ., Stanford, California.

[11] Shiffman S., Detmer W.M., Lane C.D., Fagan L.M. (1995) J Am Med Inform Assoc. 2(1):3645.

[12] Shiffman S., Lane C.D., Johnson K.B., Fagan L.M. (1992Proc Annu Symp Comput Appl Med Care, 767-71.

[13] Evett L., Tan Y.K. (2002) Disabil Rehabil. 24(11-12):607-12.

[14] Chan F.Y., Khalid H. M. (2003) Ergonomics, 46(13-14):1386-407.

[15] IBM, IBM 7772 Audio Response Unit. Form A27-271I-0 IBM Systems Library. 